\title{
Bone Marrow Aspirate and Trephine Biopsy Procedure in a Teaching and Referral Hospital in Kenya
}

\section{Teresa Lotodo ${ }^{*}$, Beatrice Melly², Christopher M. Wanjiku ${ }^{3,4}$, Caroline Kilachi ${ }^{3}$, Austin Omondi ${ }^{3}$, Japheth Kipkulei ${ }^{1}$}

\author{
${ }^{1}$ Moi University, Eldoret, Kenya \\ ${ }^{2}$ Moi Teaching and Referral Hospital, Eldoret, Kenya \\ ${ }^{3}$ AMPATH Oncology, Eldoret, Kenya \\ ${ }^{4}$ Duke University Hospital, Durham, North Carolina \\ Email: ^terrycherop@gmail.com
}

How to cite this paper: Lotodo, T., Melly, B., Wanjiku, C.M., Kilachi, C., Omondi, A. and Kipkulei, J. (2020) Bone Marrow Aspirate and Trephine Biopsy Procedure in a Teaching and Referral Hospital in Kenya. Open Journal of Blood Diseases, 10, 124-132. https://doi.org/10.4236/ojbd.2020.104014

Received: September 4, 2020

Accepted: December 22, 2020

Published: December 25, 2020

Copyright $\odot 2020$ by author(s) and Scientific Research Publishing Inc. This work is licensed under the Creative Commons Attribution International License (CC BY 4.0).

http://creativecommons.org/licenses/by/4.0/

\begin{abstract}
Introduction: Bone marrow aspiration and biopsies are carried out principally to permit cytological assessment of marrow. The procedure is also indicated for immunophenotypic, cytogenetic, molecular and other specialized investigations. The skill to perform bone marrow aspirate and biopsy is usually acquired by the medical personnel through apprenticeship during their training. The training includes performing the procedure, indications, contraindications, and associated hazards. Moi Teaching and Referral Hospital (MTRH) being a specialized Hospital in Western Kenya has several staff trained on performing the procedure. Objective: To assess the performance of bone marrow procedure by clinicians at a teaching and referral hospital. Materials and methods: A descriptive cross-sectional study was done involving 40 clinicians working in the hematology clinic and medical wards from June to December 2019. A structured questionnaire was used to collect the data and data analysis was done using frequency tables. Approval to carry out the study was sought from the institutional IRB. Results: Patients attended to were predominantly adults 33 (83\%). The superior posterior iliac crest was the commonest site for the procedure and disposable needles were routinely used in 33 (83\%) of the patients. Pain and excessive bleeding 18 (45\%) were the commonest complications associated with the procedure. Conclusion: Most of the clinicians involved doing the procedure were residents/registrars. Most of the patients attended to were adults (83\%) and majority of the clinicians performed the procedure on the posterior iliac site (96\%) using disposable needles (83\%). Local anesthesia was commonly used during the procedure $(88 \%)$. The common complications associated with the
\end{abstract}


bone marrow procedure were pain and excessive bleeding (45\%).

\section{Keywords}

Bone Marrow Aspiration, Bone Marrow Needles, Complications of Bone Marrow Procedure, Site for Bone Marrow Procedure, Trephine Biopsy

\section{Introduction}

Bone marrow aspiration and biopsies are carried out principally to permit cytological assessment but also for immunophenotypic, cytogenetic, molecular genetics, and other specialized investigations. Often, a trephine biopsy is carried out as part of the same procedure [1].

Indications for Bone marrow aspirate and Biopsy include: unexplained cytopenia, cytosis, abnormal PBF morphology suggestive of bone marrow pathology, diagnosis, staging and follow up of hematological malignancies, suspected bone marrow metastasis, unexplained organomegaly, pyrexia of unknown etiology, evaluation of iron stores, and exclusion of hematologic disease in potential allogeneic stem cell donor.

Bone marrow aspirations should be carried out by trained individuals who are aware of the indications, contraindications, and hazards of the procedure. They should follow a standard operating procedure [2]. The clinician should have made an adequate assessment of clinical and hematological features to ensure that appropriate indications exist and that all relevant tests are performed [3]. For the patient's comfort and safety, the posterior iliac crest is generally the preferred site of aspiration. Bone marrow aspiration should be preceded by evaluation of the medical history and clinical features, examination of a blood film and assessment of results of a full blood count, other laboratory tests, and radiological investigations [4].

Bone Marrow Aspirate and Biopsy is a routine procedure being carried out at Moi Teaching and Referral Hospital (MTRH). In 2016, MTRH and AMPATH oncology together with its Northern American partners set Chandaria Cancer and Chronic Disease Centre (CCCDC). It is a care and training center that provides comprehensive cancer care to patients in the region and also serves as a hands-on center for training the next generation of Kenyan cancer caregivers.

Before 2016, bone marrow procedure at MTRH was done by very few personnel including students in training. The procedure was predominantly an inpatient procedure performed in the procedure room within the medical wards for adults and children. The Bone marrow needles used then were the recycled autoclavable needles, which after sometime became blunt or chipped off. The site that was used commonly for the procedure was the sternum in adults and posterior superior iliac crest for the children. No trephine Biopsy was done then, because the needles were short in length and even smaller in diameter to acquire adequate material for processing and evaluation. Bone marrow aspirate was 
done and slides would be made for morphological assessment. No additional samples were collected for Immunophenotyping, Cytogenetics or molecular tests, though cultures for microbiological examination could be done.

Bone marrow procedure is an invasive procedure with potential complications. It is a skill that one acquires over time and samples acquired from the procedure can be used to run several tests ranging from cytology to immunophenotyping, cytogenetics, molecular and even microbiological tests. There is need to ensure that the person doing the procedure is trained, follows the SOP's and acquires adequate material for the required investigations.

Planning for the procedure is essential to ensure that there is a right indication for the procedure that the personnel required to assist are present and all the consumables for the procedure are available. The site for the procedure and the form of anesthesia to administer to the patient is also planned for. The patient should be assessed for any complications after the procedure.

The study is aimed at assessing the current practice of bone marrow aspirate and trephine biopsy procedure at the Hospital.

\section{Methods}

\section{Study Design}

A descriptive cross-sectional study was carried out at the hematology clinics and medical wards for Adults and Children. The study was carried out at MTRH, which is the second largest Referral Hospital in Kenya. Currently, the Hospital serves as a level six Hospital offering outpatient, inpatient, and specialized healthcare services including Haemato-oncology services. The Hospital serves residents of Western Kenya, parts of Eastern Uganda and Southern Sudan with a population of approximately 24 Million. In Kenya, Bone marrow procedure is mainly performed in specialized Hospitals where hematopathology services can be assessed to interpret the findings. Moi Teaching and Referral Hospital (MTRH) being a specialized Hospital in Western Kenya has several staff trained on performing the procedure.

Clinicians that perform the bone marrow procedure in the outpatient clinics and inpatient pediatric and adult wards were studied. A purposive sampling method was employed to recruit 40 participants into the study. This is because the number of clinicians who routinely perform the Bone marrow procedure are few and hence the need to recruit the ones available in the Hematology clinic, Adult and pediatric wards into the study. The Research assistant administered the questionnaire to the clinicians available during the research period.

Data was collected using questionnaires administered by trained research assistants. The study was done between October and December 2019. The information sought included, the cadre of staff performing the procedure, the age of patients whom the procedure was being undertaken, the site of procedure, type of anesthesia used, type of needle used and any complications experienced by the patient after the procedure.

The data was entered into the Statistical Package for Social Sciences (SPSS) 
version 18 and analyzed using frequency tables. The approval to carry out the Research was obtained from the Institutional IRB. Informed consent was obtained from the participants and their confidentiality was maintained.

The limitation of this study is recalling bias and the fact that doing a procedure may depend on the specific patient factors and availability of requirements which may not be consistent every time. Hence some information may not be captured using the tool employed.

\section{Result}

Figure 1 shows that most of respondents were registrars 26 (65\%). Medical officer interns $6(15 \%)$ also were involved considering that they are the $1^{\text {st }}$ on call to see the patients. They performed most of the procedures on inpatients. Over time the clinical officers $5(12.5 \%)$ in oncology unit have mastered the skill and together with the Medical officers at the unit, they are able to handle most of the outpatients. None of the consultants filled in the questionnaire.

Some clinicians performed the bone marrow aspirate on Adults 33 (63\%) and some performed it on children 7 (17\%).

Figure 2 shows that 38 (96\%) of the respondents performed the procedure at only the posterior iliac spine while about $2 \%$ at the anterior iliac spine, and $2 \%$ performed it at the sternum.

Figure 3 shows that the most frequently used needle for the BMA procedure was the one-use disposable needle at $33(83 \%), 4(10 \%)$ used disposable or autoclavable depending on availability.

Figure 4 shows that the most used type of anesthesia was local anesthesia 35 (88\%), 3 (7\%) used either local or sedation while 2 (5\%) used sedation only.

The duration taken between anesthesia administration and the procedure was reported as 3 - 5 mins in majority of the clinicians 37 (93\%). The amount of anesthesia used was $6-10 \mathrm{mls}$ in 13 (33\%) and $11-20 \mathrm{mls}$ (63\%) of the respondents.

Figure 5 shows that complication experienced after a bone marrow procedure included pain 15 (38\%), Excessive bleeding 7 (17\%), both pain and excessive bleeding accounted for 18 (45\%). There was no broken needle reported during the procedure.

\section{Discussion}

Bone marrow aspirations should be carried out by trained individuals who are aware of the indications, contraindications, and hazards of the procedure. In this study, most of the clinicians involved doing the procedure were residents/registrars $26(65 \%)$ on training. Medical officer interns 6 (15\%) also were involved considering that they are the $1^{\text {st }}$ on call to see the patients. They performed most of the procedures on inpatients. Over time the clinical officers 5 $(12.5 \%)$ in oncology unit have mastered the skill and together with the Medical officers at the unit, they are able to handle most of the outpatients.

None of the consultants filled in the questionnaire but in practice Hematolo- 
gists in the unit do the procedure, this is because they were not available to fill the questionnaire during the study. Bone marrow procedures were historically done by pathologists and hematologists and haemato-oncologists but with time this task has been shifted to other cadres. In a study by Sayed et al., looking at task Sharing and Shifting to Provide Pathology Diagnostic Service, they found out that establishing a cohort of registrars/residents, medical officers and clinical officers well trained in FNAB and BMAT biopsy procedures, would lead to increased numbers of those able to perform these procedures [5].

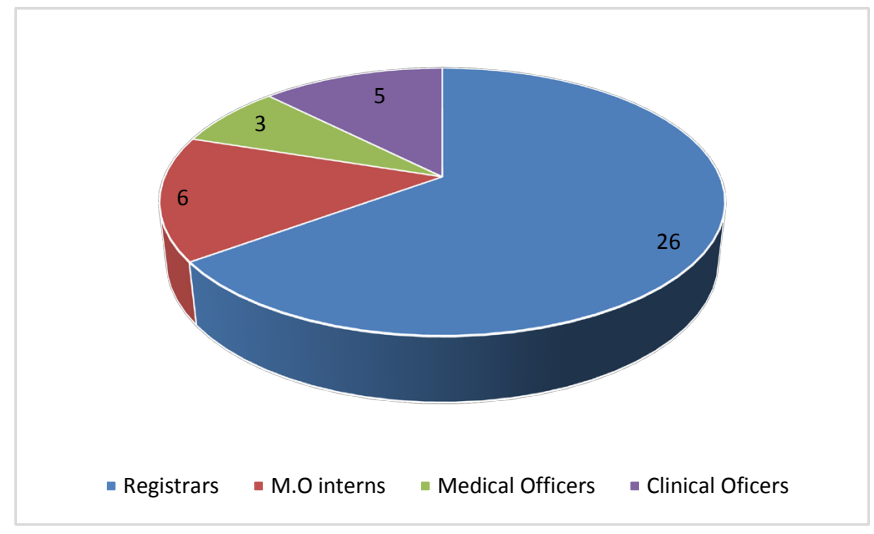

Figure 1. Pie chart showing cadre of respondents.

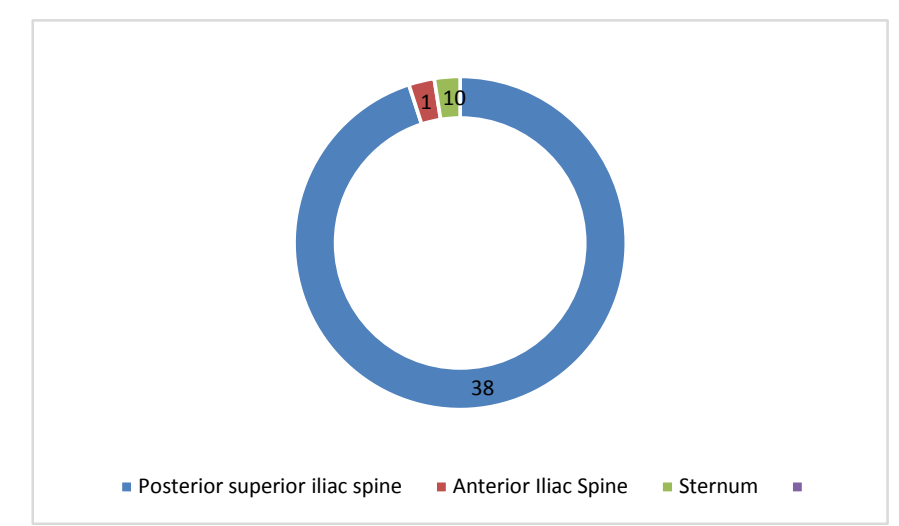

Figure 2. Pie chart showing the site of Bone marrow procedure.

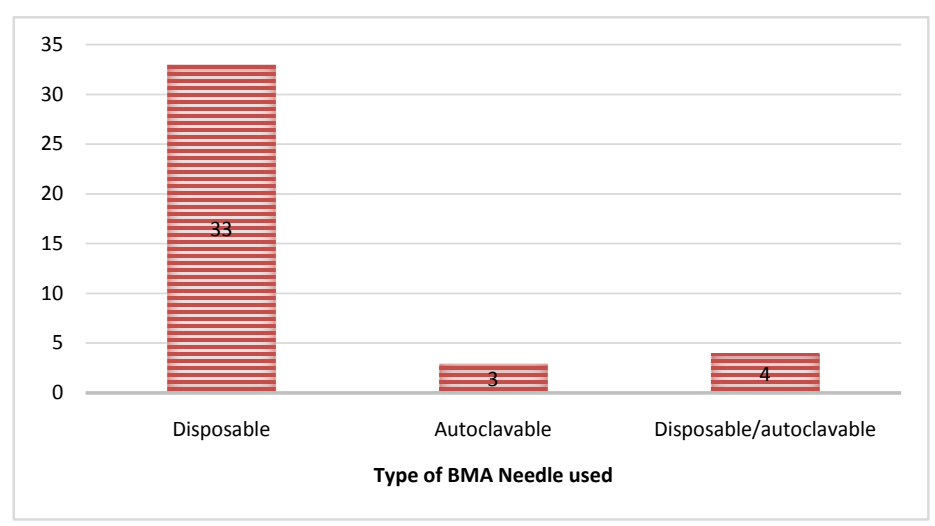

Figure 3. Type of BMA needle used. 


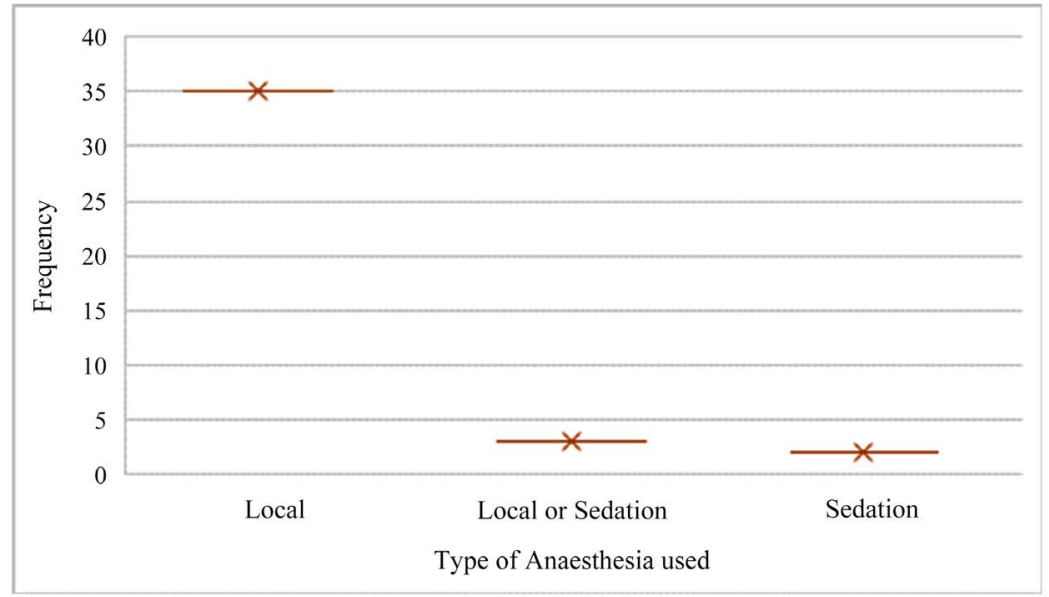

Figure 4. Type of Anesthesia used.

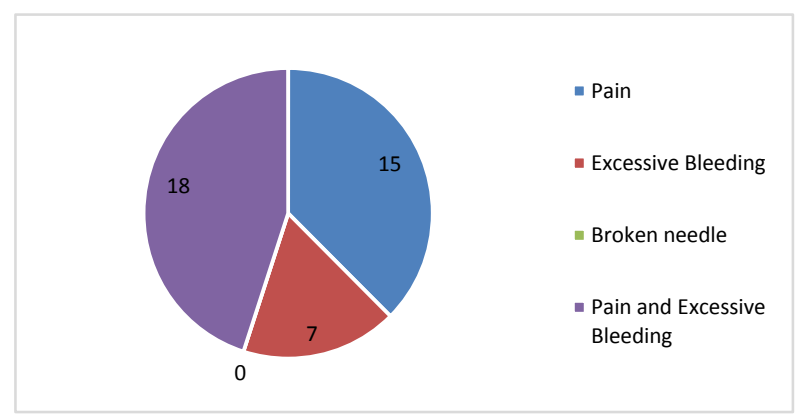

Figure 5. A Summary of Complications experienced after Bone Marrow Procedure.

A laboratory technician is often required for slide preparation as well as providing containers for the biopsy specimen [6]. In other advanced centers Ultrasound or CT technician is required to do ultrasound or CT guided aspirate at the sternum or aspirate and biopsy on the pelvic bone especially for obese patients, Asakura et al. demonstrated this in their report of an ultrasound sternal guided bone marrow aspiration [7].

There were more adults 33 (83\%) than children attended to during this study. In a similar study by Emaldhoun et al. looking at bone marrow aspiration procedure indications and diagnostic value in Northern Sudan found out that the number of adults above 15 yrs was $86 \%$ which was much higher than the pediatric population [8]. The number would vary depending on the indication.

Selection of the site for bone marrow assessment largely depends on the age of the patient and the sample to be taken. In this study, 38 (96\%) of the respondents performed the procedure at only the posterior iliac spine (Figure 2), while $2 \%$ of the respondents carried out the procedure the anterior iliac spine and $2 \%$ performed it at the sternum. According to Trejo-Ayala et al. (2015), the best site for bone marrow aspirate Trephine biopsy is the posterior superior iliac spine. It allows for easy maneuvering and less anxiety for the patient in comparison with sternum. Ultrasonography and fluoroscopy can be useful when identifying the 
biopsy site; both are particularly useful when the patient is obese [9]. If the CT-guided approach is being utilized, a radiopaque grid should be placed on the patient's skin over the iliac crest [6].

Disposable needles are preferably used than the autoclavable needles. In this study, 33 (83\%) of clinicians used only the disposable needle when carrying out the procedure, $7 \%$ used only the autoclavable and while about $10 \%$ used both needles alternately, when disposable needles were not available. This has been a big achievement, 5 yrs earlier, the only bone marrow needles available were the autoclavable ones, but currently, the disposable needles are available $90 \%$ of the time.

The sizes available are gauge 11 and 13, mainly used in adults and 15/16 mainly used in Children. The manual needle insertion procedure is the only one used in the facility. A study by Berenson et al. looked at a new method of performing bone marrow procedure using a battery powered device in comparison to manual insertion of the needle. They found out that powered device delivers larger-volume bone marrow specimens for pathology evaluation. In addition, bone marrow specimens were secured more rapidly and subjects experienced less intermediate term pain [10].

The respondents predominantly used local anesthesia $35(88 \%)$ at the site before the procedure, while only $2(5 \%)$ clinicians used sedation only (Figure 4). Adequate local anesthesia is important to minimize pain. In this study, $63 \%$ of Clinicians used between $11-20 \mathrm{mls}$ of lignocaine during local anesthesia. While about 33\% used between $6-10 \mathrm{mls}$, 2\% used between $0-5 \mathrm{mls}$ of lignocaine for the procedure. Majority of the clinicians 37 (93\%) gave a period of 3 - 5 minutes for the local anesthesia to work before the procedure was done. Some gave anti-inflammatory injectable analgesics like Tramal or Diclofenac in addition to the local anesthesia. All the pediatric patients are sedated with Ketamine and a benzodiazepine before the procedure. According to Zahid, (2015), some of the factors contributing to pain are: patients body mass index (BMI), duration of procedure and repeat procedures. Use of both pharmacological and non-pharmacological methods may reduce pain and anxiety in patients [11]. Cerchione et al., (2019) in their study found out that, the use of opioids and Benzodiazepines in addition to local anesthesia, reduced the patients anxiety and pain compared to using local anesthesia alone.

There are several complications associated with performing bone marrow procedure, Local complications like pain, bleeding and infection from puncture site are considered as minor ones. Major ones are due to improper technique or instrument usage and are as follows: manubrial-sternal dehiscence, sternal fracture, pneumopericardium, pneumothorax and biopsy needle breakage. In this study, there were some complications associated with the procedure, which patients reported to the clinician. The complications most experienced after a bone marrow procedure included pain $15(38 \%)$ and excessive bleeding in $7(17 \%)$ at site of the procedure. In a case study by Santavy et al. an $80 \mathrm{yr}$ old man developed cardiac tamponade following a sternal bone marrow aspirate [12]. In 
another study by Gendron et al. who in their study looked at pain assessment in patients undergoing bone marrow procedure using numerical pain rating scale (NPRS), found that level of anxiety, quality of the information given to the patient, operator's experience, and bone texture influenced the intensity of pain experienced by a patient [13].

\section{Conclusion}

Most of the clinicians involved doing the procedure were residents/registrars. Most of the patients attended were adults (83\%) and majority of the clinicians performed the procedure on the posterior iliac site (96\%) using disposable needles (83\%). Local anesthesia was commonly used during the procedure $(88 \%)$. The common complications associated with the bone marrow procedure were pain and excessive bleeding (45\%).

\section{Conflicts of Interest}

The authors declare no conflicts of interest regarding the publication of this paper.

\section{References}

[1] Bain, B.J. (2001) Bone Marrow Aspiration. Journal of Clinical Pathology, 54, 657-663. https://doi.org/10.1136/jcp.54.9.657

[2] Abla, O., Friedman, J. and Doyle, J. (2008) Performing Bone Marrow Aspiration and Biopsy in Children: Recommended Guidelines. Paediatrics \& Child Health, 13, 499-501. https://doi.org/10.1093/pch/13.6.499

[3] Lee, S.-H., Erber, W.N., Porwit, A., Tomonaga, M. and Peterson, L.C. (2008) ICSH Guidelines for the Standardization of Bone Marrow Specimens and Reports. International Journal of Laboratory Hematology, 30, 349-364. https://doi.org/10.1111/j.1751-553X.2008.01100.x

[4] Trejo-Ayala, R.A., Luna-Pérez, M., Gutiérrez-Romero, M., Collazo-Jaloma, J., Cedillo-Pérez, M.C. and Ramos-Peñafiel, C.O. (2015) Bone Marrow Aspiration and Biopsy. Technique and Considerations. Revista Médica del Hospital General de México, 78, 196-201. https://doi.org/10.1016/j.hgmx.2015.06.006

[5] Sayed, S., et al. (2018) Task Sharing and Shifting to Provide Pathology Diagnostic Services: The Kenya Fine-Needle Aspiration Biopsy Cytology and Bone Marrow Aspiration and Trephine Biopsy Training Program. JCO Global Oncology, 4. https://doi.org/10.1200/JGO.18.00094

[6] Rindy, L.J. and Chambers, A.R. (2020) Bone Marrow Aspiration and Biopsy. In StatPearls. Treasure Island (FL): StatPearls Publishing.

[7] Asakura, Y., Kinoshita, M., Kasuya, Y., Sakuma, S. and Ozaki, M. (2017) Ultrasound-Guided Sternal Bone Marrow Aspiration. Blood Research, 52, 148-150. https://doi.org/10.5045/br.2017.52.2.148

[8] Elmadhoun, W.M., Noor, S.K., Bushara, S.O., Almobarak, A.O., Husain, N.E. and Ahmed, M.H. (2015) Bone Marrow Aspiration in North Sudan: The Procedure, Indications and the Diagnostic Value. International Journal of Health Sciences, 9, 434-439. https://doi.org/10.12816/0031233

[9] Ridgeway, J.A., Tinsley, S. and Kurtin, S.E. (2017) Practical Guide to Bone Marrow 
Sampling for Suspected Myelodysplastic Syndromes. Journal of the Advanced Practitioner in Oncology, 8, 29-39. https://doi.org/10.6004/jadpro.2017.8.1.3

[10] Berenson, J.R., et al. (2011) Using a Powered Bone Marrow Biopsy System Results in Shorter Procedures, Causes Less Residual Pain to Adult Patients, and Yields Larger Specimens. Diagnostic Pathology, 6, 23-23.

https://doi.org/10.1186/1746-1596-6-23

[11] Zahid, M.F. (2015) Methods of Reducing Pain during Bone Marrow Biopsy: A Narrative Review. Annals of Palliative Medicine, 4, 184-193.

[12] Santavy, P., Troubil, M. and Lonsky, V. (2013) Pericardial Tamponade: A Rare Complication of Sternal Bone Marrow Biopsy. Hematology Reports, 5, No. 3. https://doi.org/10.4081/hr.2013.e13

[13] Gendron, N., et al. (2019) Pain Assessment and Factors Influencing Pain during Bone Marrow Aspiration: A Prospective Study. PLoS ONE, 14, No. 8.

https://doi.org/10.1371/journal.pone.0221534 